\title{
ICT and Value Sensitive Design
}

\author{
Jeroen van den Hoven \\ Extraordinary Professor and Associate professor \\ Department of Philosophy \\ Delft University of Technology - The Netherlands \\ m.j.vandenhoven@tbm.tudelft.nl
}

\begin{abstract}
It is argued that information systems are intentionally or unintentionally informed by moral values of their makers. Since information technology has become a constitutive technology which shapes human life it is important to be aware of the value ladenness of IT design. Examples of values incorporated in design are discussed. The approach to software engineering and systems development, which is referred to as "value sensitive design", studies the ways in which our accepted moral values can be operationalized and incorporated in IT design. The historical background and future program of value sensitive design in IT are sketched.
\end{abstract}

Keywords: ICT, Ethics, Value Sensitive Design, Value Laden IT

In the age of high technology and applied science it seems appropriate to think about how to behave morally with information technology: how to prevent harm to others, to improve the quality of life and to solve some of our hardest social problems. I sketch a conception of doing responsible information technology. This approach is sometimes referred to as Value Sensitive Design. Value Sensitive Design is a way of engaging ICT that aims at making moral values part of technological design, research and development. It assumes that human values, norms, moral considerations can be imparted to the things we make and use and it construes information technology (and other technologies for that matter) as a formidable force which can be used to make the world a better place, especially when we take the trouble of reflecting on its ethical aspects in advance.

The idea of Value Sensitive Design has a wider application in all engineering and design disciplines, but was first proposed and discussed in connection with information and communication technology and that is still its main area of application. There were several ideas and proponents that gave rise to it. First, there 
is work by researchers such as Terry Winograd, Batya Friedman ${ }^{1}$, John Perry, Ben Shneiderman and Helen Nissenbaum ${ }^{2}$ in the mid nineties ${ }^{3}$. They showed that software could easily come to contain biases, arbitrary assumptions and peculiar worldviews of makers, which could affect users in various ways. They also stressed that systems could be designed in compliance with ethical ideals such as privacy and user autonomy. Secondly, legal scholars around the same time observed that regulation in society was taking place by means of computer code and software. Code functioned as law and laws would in the future literally be en-coded, as Joel Reidenberg and Larry Lessig ${ }^{4}$ pointed out. Advocates of so-called Privacy Enhancing Technology at the Dutch and Canadian Data Protection Offices ${ }^{5}$ observed that this was probably the only way in which we could deal with privacy compliance and law enforcement issues given the increasing amount of laws and regulation and the vast amount of data that are processed in our society. It is impossible to have lawyers check manually whether certain data practices are in breach or in compliance with the law. The software would in the long run have to take care of that on our behalf, and not only in the privacy area.

Rob Kling's Social Informatics ${ }^{6}$ had been instrumental in making research in social studies in science and technology available in the ICT field and highlighted the social shaping of technology. At Rensselaer Polytechnic Langdon Winner ${ }^{7}$ famously argued that artefacts can be used for political purposes and in important ways contain the ideals of their makers and Deborah Johnson had articulated a broad range of ethical issues in computing ${ }^{8}$. These developments converged in a focus on moral values and IT design.

The resulting idea is straightforward: Information technology has become a constitutive technology and partly constitutes the things to which it is applied. It shapes our discourses, practices and institutions and experiences in important ways. What health care, public administration, politics, education, science, transport and logistics are and will be within twenty years from now, which values will be expressed in it, will in important ways be determined by the ICT applications we decide to use in these domains.

A couple of examples will demonstrate how intimate the relation between IT and moral values often is.

Jenkins and McCauley (2006) describe a software application where the choice for an algorithm has drastic and far reaching moral consequences ${ }^{9}$. In their paper "GIS, SINKS, FILL, and disappearing wetlands: unintended consequences in algorithm development and use" they describe how Geographic Information Systems

1See for an overview of work and projects:

http://www.ischool.washington.edu/vsd/publications.html

2 See for an overwview: http://www.nyu.edu/projects/valuesindesign/index.html

3 See for example also http://www.designforvalues.org/projects.htm

4 For information about Larry Lessig's work: http://www.lessig.org/blog/

5 See for an explanation of PET by one of Privacy researchers who coined the term: $\mathrm{http}: / / \mathrm{www}$.datenschutz.de/files/what is_pet.htm

$6 \mathrm{http}: / / \mathrm{www}$.slis.indiana.edu/St/si200̄1. $\overline{\mathrm{html}}$

7 Winner, Langsdon. "Do Artefacts Have Politics?" Daedalus 109 (1980): 121-36.

8 Deborah Johnson, Computer Ethics, Prentice Hall, 1985, 1994.

9 Jenkins and McCauley, paper at SAC2006, ACM conference, Dijon. 
(GIS) software has become an important computational tool in several fields. Based on the output from this software GIS users make important decisions to plan and manage landscapes (e.g., cities, parks, forests) with real consequences for the ecosystems. Jenkins and McCauley discuss a programming decision in a GIS algorithm originally used to discern flow direction in hydrological modelling: the mapping of streams and rivers. Topographic depressions ("sinks") are "filled" in the algorithm to map water flow downstream; otherwise, the GIS algorithm cannot solve the problem of accurately calculating and representing the flow direction. Unfortunately, sinks are often "isolated" wetlands which provide essential habitat for many species not commonly found elsewhere. Thus the algorithmic filling of sinks can make these wetlands "disappear" in GIS output and land-use decisions based on this output. This outcome occasioned by the choice of the algorithm may have potentially devastating real-world consequences for numerous wetlands because land-use plans made in ignorance cannot adequately conserve these unique habitats and the vital ecosystem services that wetlands provide. These consequences were not anticipated by the programmers who originally implemented the flow direction algorithm and may not be known to GIS users. Local authorities and planners however may welcome this particular effect, since it would make environmentally contested projects invisible and would give environmentalists no occasion to protest.

Dardelet and Darcy describe the development and testing of software that supports firemen in their operations by means of a real time broadband audio videolink with an emergency medicine centre ${ }^{10}$. The software and hardware seemed to work perfectly well in the testing situations, from a technical point of view. But one thing which was not anticipated and turned out to be of crucial importance for the success of this type of application was the clarity and consensus about professional roles and responsibilities of firemen and medical professionals. Tests clearly demonstrated that there were conflicting views of what had to be done by the firemen on the site of the fire, accident or disaster. Emergency medicine experts have different priorities from their professional ethics than firefighters. Different value systems and different conceptions of what is important and relevant, different ideas about responsibility meet and the results are often confusion and disagreement. A value analysis needs to be made in advance and protocols need to implement them. No IT application of this type can work satisfactorily if its value implementation is inadequate.

Other work by e.g. Nissenbaum and Camp clearly illustrates how values choice inform the design, coding, and architecture of systems and applications.

If our moral and political discourse on user autonomy, patient centred-ness and citizen centred-ness, our privacy, security is to be more than an empty promise, these values will have to be expressed in the design, architecture and specifications of systems. If we want our information technology - and the use that is made of it - to be just, fair and safe, we must see to it that it inherits our good intentions. Moreover it must be seen to have those properties, we must be able to demonstrate that they possess these morally desirable features, compare different applications from these

10 B. Dardelet and S. Darcy, Rescuing the Emergency - Multiple Expertise and IT in the Emergency field.Methods of Information in Medicine, 2003, 42, 4, pp. 353-359. 
value perspectives and motivate political choices and justify investments from this perspective.

Value Sensitive Design provides us with the opportunity to deal with these ethical issues in a new and fresh way: by 'front-loading ethics' and by means of the pro-active integration of ethical reflection in the stage of design of architectures, requirements, specifications, standards, protocols, incentive structures, and institutional arrangements.

An ethics of information technology should open the black box of technology design and development and describe its rich and heterogeneous content, and make an inventory of the degrees of freedom in the design and engineering process, which choices have been made and can be made, preferably before the problem becomes manifest, preferably before it is too late, preferably when ethics can still make a difference.

Moral philosophers also need to rise to the occasion, not only IT professionals, software engineers and computer scientists. They not only have a tendency to forget about the way technology sets the stage for our actions in almost every sector of society and every department of our lives in transport, housing, education, telecommunication, food, energy, public administration, health care, but they have also failed to see how intimately technology and the good life are connected.

Technology should not be construed simply as a necessary condition for our survival, but Technology always aims at making life slightly more comfortable, more easy, less cold, less hungry, less painful. In principle we could do without technology in many cases without immediately perishing. The idea that somehow we could not survive without the fire, the flint stone arrowhead, clothing, cars and fossil fuel, lasagne in deep freeze and magnetrons, is of course plainly false. We could survive: our lives would be nasty, cold and uncomfortable, less convenient. Every technological device, every gadget, comes with the implicit suggestion that life would be more agreeable if we started to use it. This applies to the thumbscrew, syringe, roller skates and razor blades. Those who make the suggestion for the invention and use of these artefacts can be - and sometimes are - mistaken, wicked, or confused (or a combination of those), but in any case their suggestion should above all be evaluated as a contribution to the good life, since the good life is the terminus ad quem of technology. The first question which should therefore be asked with respect to technology is whether it actually delivers the goods, whether it really contributes to the good life, however conceived.

\section{Values and Design: at the crossroads}

Ethics has seen some changes in the course of the last 100 years. It started in the beginning of the $20^{\text {th }}$ century as a predominantly analytical and meta-ethical enterprise. Later ordinary language philosophers arrived on the scene and continued the work with different means. In the sixties however the philosophical climate changed. Ethics witnessed its "Applied Turn", a turn to practice and context. Especially in the USA philosophers started to realize that philosophy could contribute to social and political debates about the Vietnam War and civil rights, 
later on abortion and euthanasia, by clarifying notions and structuring arguments. Ever since the sixties applied ethics has been growing. Every conceivable profession and cluster of societal issues has a special or applied ethics named after itself, from library ethics to sports ethics. I think we now are slowly moving into a third phase where not only application of moral theories and applied analysis is considered a legitimate and important activity, but design questions start to make their appearance.

The work of the Harvard philosopher John Rawls' is one of the first that gave rise to talk about design in ethics. Thinking about social justice could in the context of his theory be described as formulating and justifying the principles of justice in accordance with which we should design the basic institutions in society.

Contemporary ethicists (such as Thomas Pogge, Bob Goodin, Dennis Thompson) do not only want to offer an applied analysis, they also want to think about some of the real world conditions, institutions and incentive structures that need to be realized if applied analyses are to stand a chance in their implementation. This "design turn" in applied ethics is still focussed on institutional design, but the second stage will most certainly also bring into view the design of technology, technological artefacts and socio-technical systems.

An interesting positive parallel development can be observed in ICT, and probably also in other engineering disciplines: a shift from technology simpliciter, to technology in context. In the first phase of its development in the sixties and seventies the social and organizational context did not matter much in the production of ICT applications. Hardly anyone bothered to ask about human users, use and usability and the fit with the organisational and social context. Computers were a new and fascinating technology: solutions looking for problems. In the second stage of the development in the eighties and nineties -after many failed projects, worthless applications and bad investments- one gradually started to realize that there were human users, with real needs and real desires and real organizations with peculiar properties. It started to occur to many at that time that it would be wise and profitable to try and accommodate user requirements, conditions on the work floor in the early stages of the development of applications. The social and behavioural sciences came to the aid of ICT in this period. But this is still a minimal way of taking the needs, interests and of users, organizations and society into account, namely as mere constraints on the successful implementation of systems.

If I am not mistaken we are now entering a third phase in the development of ICT, namely one where the needs of human users, the values of citizens, patients, and some of our social questions are considered in their own right and are starting to drive research and development of ICT. ${ }^{11}$

We are at the intersection of both developments: the increasing interest in applied ethics for the design perspective and the increasing interest in technology for value aspects of design. If I am not mistaken this historical situation provides a

11 One of the interesting examples of that approach to date is the Californian Institute CITRIS (Centre for IT Research in the Interest of Society) endowed with 320 million US dollar. The CITRIS research agenda is determined by social problems and their solution. See http://www.citris.berkeley.edu/ 
favourable condition for the further development of Value Sensitive Design research and to do good by building what is good. 